\title{
On Maps of Period 2 on Prime and Semiprime Rings
}

\author{
H. E. Bell ${ }^{1}$ and M. N. Daif ${ }^{2}$ \\ ${ }^{1}$ Department of Mathematics, Brock University, St. Catharines, ON, Canada L2S 3A1
}

${ }^{2}$ Department of Mathematics, Al-Azhar University, Nasr City, Cairo 11884, Egypt

Correspondence should be addressed to M. N. Daif; nagydaif@yahoo.com

Received 29 March 2014; Accepted 16 August 2014; Published 22 September 2014

Academic Editor: Ram N. Mohapatra

Copyright ( 2014 H. E. Bell and M. N. Daif. This is an open access article distributed under the Creative Commons Attribution License, which permits unrestricted use, distribution, and reproduction in any medium, provided the original work is properly cited.

A map $f$ of the ring $R$ into itself is of period 2 if $f^{2}(x)=x$ for all $x \in R$; involutions are much studied examples. We present some commutativity results for semiprime and prime rings with involution, and we study the existence of derivations and generalized derivations of period 2 on prime and semiprime rings.

\section{Introduction}

Let $R$ be a ring with center $Z=Z(R)$, and for each $x, y \in R$, let $[x, y]$ denote the commutator $x y-y x$. Note that $[x y, w]=$ $x[y, w]+[x, w] y$ and $[w, x y]=x[w, y]+[w, x] y$ for all $x, y, w \in R$-facts we will use repeatedly.

Let $S$ be a nonempty subset of $R$. A map $f: R \rightarrow R$ is said to be of period 2 on $S$ if $f^{2}(x)=x$ for all $x \in S$, and $S$ is called an $f$-subset if $f(S)=S$. If $[x, f(x)]=0$ for all $x \in S$, then $f$ is said to be commuting on $S$; if $[x, y]=[f(x), f(y)]$ for all $x, y \in S$, then, as in [1], $f$ is called strong commutativitypreserving on $S$.

We assume the reader is familiar with the definitions of derivation and involution. We define an additive map $F$ : $R \rightarrow R$ to be a right (resp. left) generalized derivation on $R$ if $F(x y)=F(x) y+x d(y)$ (resp., $F(x y)=d(x) y+x F(y))$ for all $x, y \in R$, where $d$ is a derivation on $R$, called the associated derivation. If $F$ is both a right generalized derivation and a left generalized derivation with the same associated derivation, we call $F$ a generalized derivation. (Note that this definition is different from that of Hvala in [2]; his generalized derivations are our right generalized derivations.)

Our purpose is to study existence and properties of involutions, derivations, and generalized derivations of period 2 on certain subsets of semiprime and prime rings.

\section{Two Commutativity Results for Rings with Involution}

There are several known commutativity results for rings with involution (cf. [3, Chapter 3]). We now present a result showing the equivalence of two commutativity conditions on a $*$-ideal of a semiprime ring with involution.

Theorem 1. Let $R$ be a semiprime ring with involution $*$, and let $U$ be $a *$-ideal of $R$. Then $*$ is commuting on $U$ if and only if $*$ is strong commutativity-preserving on $U$.

Proof. Assume first that $*$ is commuting on $U$; that is, $\left[x, x^{*}\right]=0$ for all $x \in U$. By linearizing we get

$$
\left[x, y^{*}\right]=\left[x^{*}, y\right] \quad \forall x, y \in U .
$$

It follows that $\left[x y, x^{*}\right]=\left[(x y)^{*}, x\right]$ and hence $x\left[y, x^{*}\right]=$ $\left[y^{*}, x\right] x^{*}$, and by (1) we get

$$
x\left[y^{*}, x\right]=\left[y^{*}, x\right] x^{*} \quad \forall x, y \in U .
$$

Since $U$ is a $*$-ideal, (2) yields

$$
x[x, y]=[x, y] x^{*} \quad \forall x, y \in U .
$$

Substituting $z y$ for $y, z \in U$, we now get $x[x, z y]=[x, z y] x^{*}$, so that $x z[x, y]+x[x, z] y=[x, z] y x^{*}+z[x, y] x^{*}$. Using 
(3) to replace the last term in this equation by $z x[x, y]$ and the second term by $[x, z] x^{*} y$, we see that $[x, z][x, y]+$ $[x, z]\left[x^{*}, y\right]=0$, so by $(1)$,

$$
[x, z]\left[x, y+y^{*}\right]=0 \quad \forall x, y, z \in U .
$$

Replacing $z$ by $z w$ yields $[x, z] U\left[x, y+y^{*}\right]=\{0\}$ for all $x, y \in$ $U$, so $\left[x, y+y^{*}\right] U\left[x, y+y^{*}\right]=\{0\}$ for all $x, y \in U$. Since an ideal of a semiprime ring is a semiprime ring, we conclude that

$$
\left[x, y+y^{*}\right]=0 \quad \forall x, y \in U .
$$

Now (5) may be rewritten as

$$
[x, y]=\left[y^{*}, x\right]
$$

so by replacing $x$ by $x^{*}$ we get $\left[x^{*}, y\right]=\left[y^{*}, x^{*}\right]$. But by (6) $\left[x^{*}, y\right]=[y, x]$; hence $[y, x]=\left[y^{*}, x^{*}\right]$ for all $x, y \in U$, so that $*$ is strong commutativity-preserving on $U$.

For the converse, we assume that $*$ is strong commutativity-preserving on $U$, which means that $[x, y]=\left[x^{*}, y^{*}\right]$ for all $x, y \in U$. Substituting $x y$ for $y$, we get

$$
x[x, y]=[x, y] x^{*} \quad \forall x, y \in U
$$

This is just equation (3), so we may argue as before that

$$
\left[x, y+y^{*}\right]=0 \quad \forall x, y \in U,
$$

and $y+y^{*} \in Z(U)$ for all $y \in U$. It follows at once that $\left[y, y^{*}\right]=0$ for all $y \in U$; that is, $*$ is commuting on $U$.

The proof just given yields a result for prime rings with involution. Before stating our theorem, we mention that we are using the symbols $S$ and $K$ to denote the sets of symmetric elements and skew elements, respectively, in the ring $R$ with involution $*$.

Theorem 2. Let $R$ be a prime ring with involution $*$, with $\operatorname{char}(R) \neq 2$. If $*$ is commuting on some nonzero $*$-ideal $U$, then $S \subseteq Z$.

Proof. It follows from (5) that if $y \in S \cap U$, then $y \in Z(U)$, and since in a prime ring the center of a nonzero ideal is contained in the center of $R, S \cap U \subseteq Z(R)$. Suppose that $S \cap U \neq\{0\}$, and let $z \in(S \cap U) \backslash\{0\}$. Then for any $s \in S, s z \in U \cap S$, so $s z \in Z(R)$. Since $z$ is not a zero divisor, we get $s \in Z(R)$.

To complete the proof, we need only show that $S \cap U \neq$ $\{0\}$. Suppose, on the contrary, that $S \cap U=\{0\}$. Then for any $y \in U, y+y^{*}=0$; hence $U \subseteq K$. But for any $k \in K, k^{2} \in S$; therefore $y^{2}=0$ for all $y \in U$, and we have contradicted a well-known result of Levitzki [4, Lemma 1.1].

\section{On Nonexistence of Derivations of Period 2}

If $R$ is an algebra over $G F(p)$ with trivial multiplication, the map given by $d(x)=(p-1) x$ is a derivation of period 2 . We do not know whether there exist less obvious examples.

Clearly any derivation $d$ of period 2 must be a bijection, so there exists no $c \neq 0$ such that $d(c)=0$. It follows that a ring $R$ with 1 admits no derivation which is of period 2 on $R$.
There do exist semiprime rings $R$ admitting a derivation which is a bijection, for example, the $\mathbb{R}$-algebra with basis $\left\{e^{k x} \mid k=1,2, \ldots\right\}$, with $d$ being the usual differentiation. Obviously this example is not of period 2, and we will show that a semiprime ring admits no derivation of period 2 on $R$.

Theorem 3. Let $R$ be a semiprime ring and $U$ a nonzero right ideal. Then $R$ admits no derivation $d$ such that $d$ is of period 2 on $U$.

Proof. Suppose there exists a derivation $d$ on $R$ such that $d^{2}(x)=x$ for all $x \in U$. For $x, y \in U, x d(y) \in U$ and the condition that $x d(y)=d^{2}(x d(y))$ yields

$$
x d(y)+2 d(x) y=0 \quad \forall x, y \in U .
$$

Since $d^{2}(x y)=x y=d(d(x) y+x d(y))$, we get

$$
x y+2 d(x) d(y)=0 \quad \forall x, y \in U
$$

and replacing $y$ by $y r$ in (10), we obtain

$$
2 d(x) y d(r)=0 \quad \forall x, y \in U, r \in R .
$$

Substituting $r x$ for $r$ in (11), we get $2 d(x) \operatorname{yrd}(x)=0$; hence

$$
2 d(x) y R d(x) y=\{0\} \quad \forall x, y \in U .
$$

But $R$ is semiprime, so $2 d(x) y=0$ for all $x, y \in U$, and by (9),

$$
x d(y)=0 \quad \forall x, y \in U .
$$

Therefore

$$
\begin{aligned}
d(x d(y)) & =d(x) d(y)+x d^{2}(y) \\
& =d(x) d(y)+x y=0 \quad \forall x, y \in U,
\end{aligned}
$$

which together with (10) yields $x y=0$ for all $x, y \in U$. In particular, $x^{2}=0$ for all $x \in U$, contrary to Levitzki's result.

Corollary 4. A semiprime ring $R$ admits no derivation of period 2 on $R$.

Remark 5. Of course any derivation $d$ of period 2 on $R$ satisfies $d^{3}=d$. It is shown in [5] that a noncommutative semiprime ring, though it has no derivations of period 2, may have many nonzero derivations for which $d^{3}=d$; for any noncentral idempotent $e$, the inner derivation $d_{e}$ is an example.

\section{Generalized Derivations of Period 2}

Any ring admits right generalized derivations of period 2, namely, the identity map and its negative. Moreover, if $R$ has 1 and $c \in R$ with $c^{2}=1$, then $F(x)=c x$ defines a right generalized derivation of period 2 . We show that, in many prime rings, there are no other possibilities. 
We will make use of several easy lemmas.

Lemma 6. Let $R$ be an arbitrary ring. If $F$ is a generalized derivation on $R$, then $F(Z) \subseteq Z$.

Proof. Let $x \in R$ and $z \in Z$. Then $F(z x)=F(x z)$, so that $F(z) x+z d(x)=d(x) z+x F(z)$, where $d$ is the associated derivation of $F$. Since $[z, d(x)]=0$, the result follows at once.

Lemma 7. Let $R$ be a prime ring with $\operatorname{char}(R) \neq 2$, and let $d$ be a derivation on $R$. If the right generalized derivation given by $F(x)=x+d(x)$ (resp., $F(x)=-x+d(x)$ ) for all $x \in R$ is of period 2 on $R$, then $F$ is the identity map (resp., the negative of the identity map) on $R$.

Proof. Consider the case $F(x)=x+d(x)$ for all $x \in R$. If $F$ is of period 2, we have $x=F^{2}(x)=F(x+d(x))=F(x)+F(d(x))=$ $x+d(x)+d(x)+d^{2}(x)$; hence

$$
2 d(x)+d^{2}(x)=0 \quad \forall x \in R .
$$

Replacing $x$ by $x y$, we get $2(d(x) y+x d(y))+d^{2}(x) y+$ $2 d(x) d(y)+x d^{2}(y)=0$; that is, $\left(2 d(x)+d^{2}(x)\right) y+x(2 d(y)+$ $\left.d^{2}(y)\right)+2 d(x) d(y)=0$ for all $x, y \in R$. In view of (15) and the assumption that $\operatorname{char}(R) \neq 2$, this equation gives

$$
d(x) d(y)=0 \quad \forall x, y \in R .
$$

It is well known and easy to prove that if $R$ is prime and $d$ is a nonzero derivation, then $a \in R$ and $a d(R)=\{0\}$ implies $a=0$. Thus, from (16) we conclude that $d=0$ and therefore $F$ is the identity map on $R$. A similar argument works if $F(x)=$ $-x+d(x)$ for all $x \in R$.

Lemma 8. Let $R$ be a prime ring with $\operatorname{char}(R) \neq 2$, and let $F$ be a right generalized derivation on $R$ with associated derivation d. If $F$ is of period 2 on $R$, then $d(Z)=\{0\}$.

Proof. For all $x, y \in R, x y=F^{2}(x y)=F(F(x) y+x d(y))=$ $F^{2}(x) y+F(x) d(y)+F(x) d(y)+x d^{2}(y)$; hence

$$
2 F(x) d(y)+x d^{2}(y)=0 \quad \forall x, y \in R .
$$

Replacing $x$ by $F(x)$ in (17) yields

$$
2 x d(y)+F(x) d^{2}(y)=0 \quad \forall x, y \in R .
$$

Letting $z \in Z$ and $x \in R$ and replacing $x$ by $x z$ in (18), we get $2 x z d(y)+(F(x) z+x d(z)) d^{2}(y)=0=z(2 x d(y)+$ $\left.F(x) d^{2}(y)\right)+d(z) x d^{2}(y)$, so by (18) we obtain

$$
d(z) R d^{2}(y)=\{0\} \quad \forall y \in R .
$$

If $d(Z) \neq\{0\}$, we conclude that $d^{2}=0$. But since $R$ is prime and $\operatorname{char}(R) \neq 2$, it is easy to show that $d \neq 0$ implies $d^{2} \neq 0$; hence $d(Z)=\{0\}$ as claimed.

Theorem 9. Let $R$ be a (not necessarily commutative) domain with 1 , with char $(R) \neq 2$. If $F$ is a right generalized derivation on $R$ of period 2, then $F$ is the identity map or its negative.
Proof. Note that

$$
F(x)=F(1 x)=F(1) x+d(x) \quad \forall x \in R .
$$

Taking $x=1$ in (17) and (18) and letting $c=F(1)$, we have $2 c d(y)+d^{2}(y)=0$ and $2 d(y)+c d^{2}(y)=0$ for all $y \in R$. It follows that $2 d(y)+c(-2 c d(y))=0$; that is, $\left(2-2 c^{2}\right) d(y)=0$; hence

$$
\left(c^{2}-1\right) d(y)=0 \quad \forall y \in R .
$$

If $d \neq 0$, we have $c=1$ or $c=-1$, so that $F(x)=x+d(x)$ for all $x \in R$ or $F(x)=-x+d(x)$ for all $x \in R$. But by Lemma 7 this would imply $d=0$; hence $d=0$ and

$$
F(x)=c x \quad \forall x \in R
$$

Since $F$ is of period 2, $x=c^{2} x$ and hence $\left(c^{2}-1\right) x=0$ for all $x \in R$. Thus, $c=1$ or $c=-1$, so by (22), $F$ is the identity map or its negative.

Corollary 10. Let $R$ be a commutative integral domain with $\operatorname{char}(R) \neq 2$. If $F$ is a right generalized derivation of period 2 on $R$, then $F$ is the identity map or its negative.

Proof. If $R$ has 1 , the result is immediate from Theorem 9. If $R$ does not have 1 , define $\widehat{F}$ on the field of fractions $K$ by $\widehat{F}(a / b)=F(a) / b$. Using the fact that $d=0$ by Lemma 8 , we can show that $\widehat{F}$ is well defined and is a right generalized derivation on $K$. By Theorem $9, \widehat{F}$ is the identity map or its negative on $K$ and it follows that $F$ is the identity map or its negative on $R$.

Theorem 11. Let $R$ be a prime ring with $Z \neq\{0\}$ and with $\operatorname{char}(R) \neq 2$. If $F$ is a generalized derivation of period 2 on $R$ with associated derivation $d$, then $F$ is the identity map or its negative.

Proof. By Lemma 6, $F(Z) \subseteq Z$; hence $F$ restricts to a generalized derivation on $Z$. Since the center of a prime ring is a commutative domain, it follows from Corollary 10 that $F(z)=z$ for all $z \in Z$ or $F(z)=-z$ for all $z \in Z$. If the former holds, then $F(z x)=F(x z)$, together with the fact that $d(Z)=\{0\}$, gives $z(F(x)-x-d(x))=0$ for all $x \in R$ and $z \in Z$. Taking $z \neq 0$ gives $F(x)=x+d(x)$ for all $x \in R$, so by Lemma $7, F$ is the identity map on $R$. A similar argument shows that if $F(z)=-z$ for all $z \in Z, F$ is the negative of the identity map.

\section{Conflict of Interests}

The authors declare that there is no conflict of interests regarding the publication of this paper.

\section{References}

[1] H. E. Bell and M. N. Daif, "On commutativity and strong commutativity-preserving maps," Canadian Mathematical Bulletin, vol. 37, no. 4, pp. 443-447, 1994. 
[2] B. Hvala, "Generalized derivations in rings," Communications in Algebra, vol. 26, no. 4, pp. 1147-1166, 1998.

[3] I. N. Herstein, Rings with Involution, University of Chicago Press, Chicago, Ill, USA, 1976.

[4] I. N. Herstein, Topics in Ring Theory, Lecture Notes in Mathematics, University of Chicago, 1965.

[5] H. E. Bell and L. C. Kappe, "Rings in which derivations satisfy certain algebraic conditions," Acta Mathematica Hungarica, vol. 53, no. 3-4, pp. 339-346, 1989. 


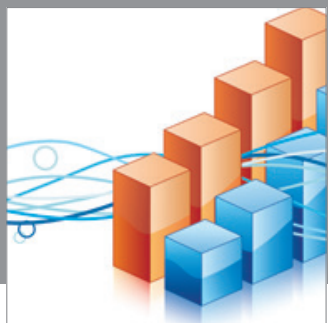

Advances in

Operations Research

mansans

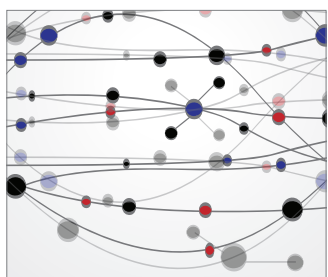

The Scientific World Journal
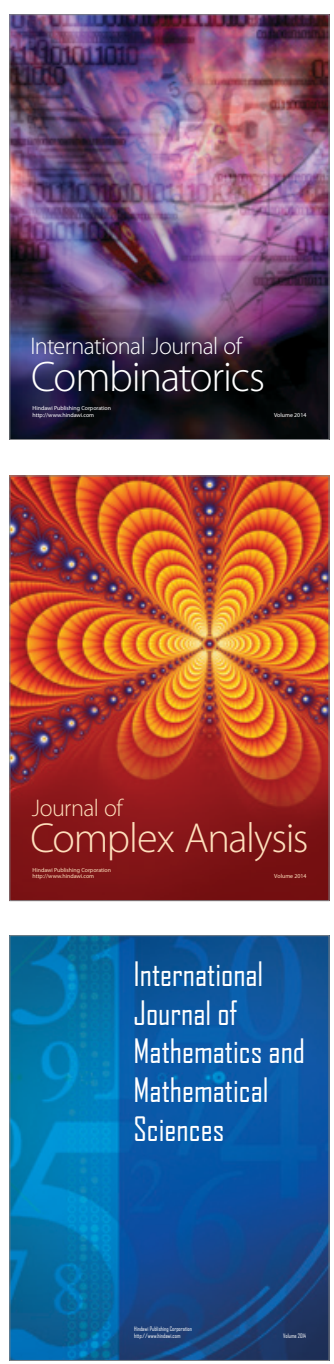
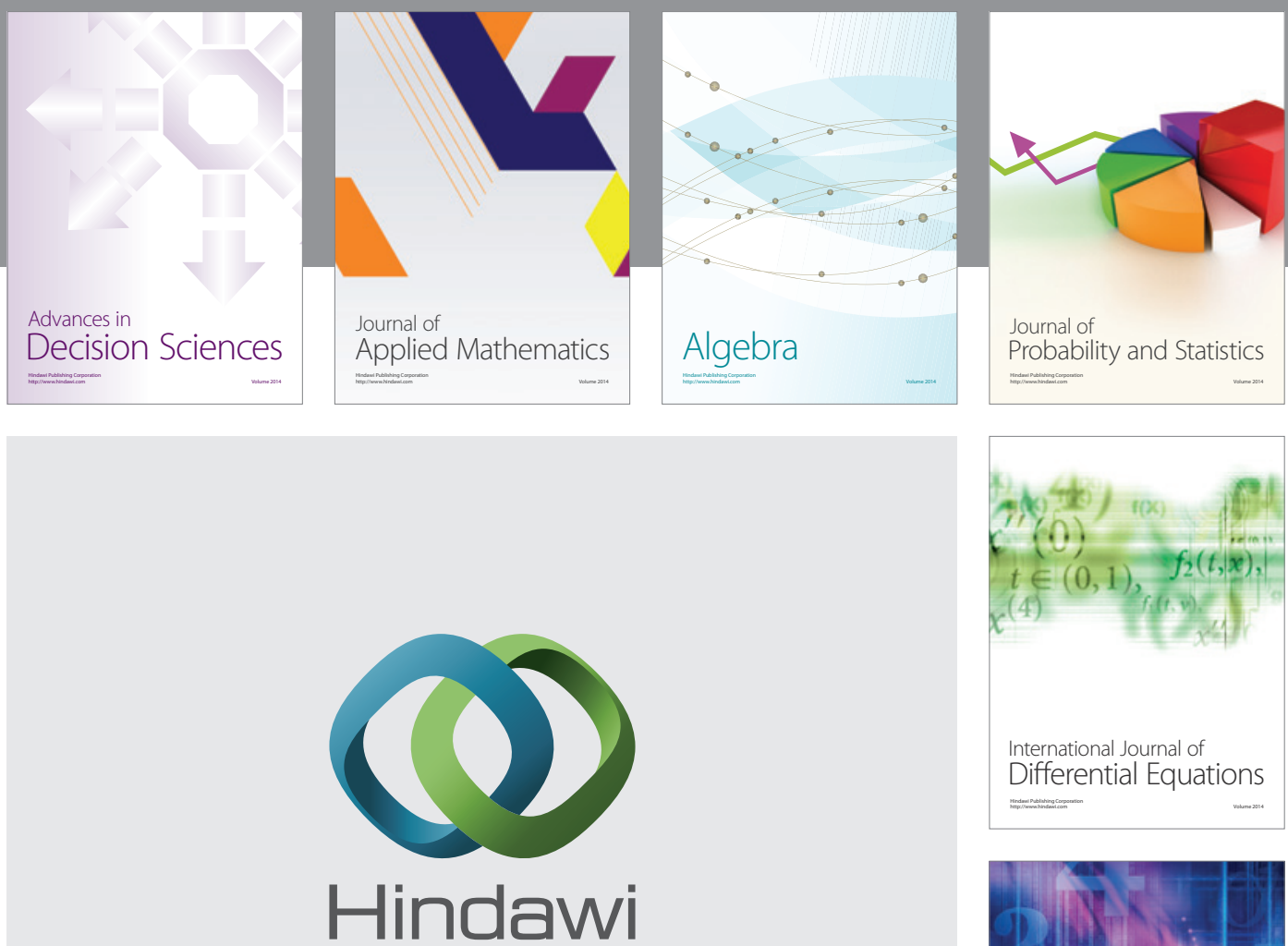

Submit your manuscripts at http://www.hindawi.com
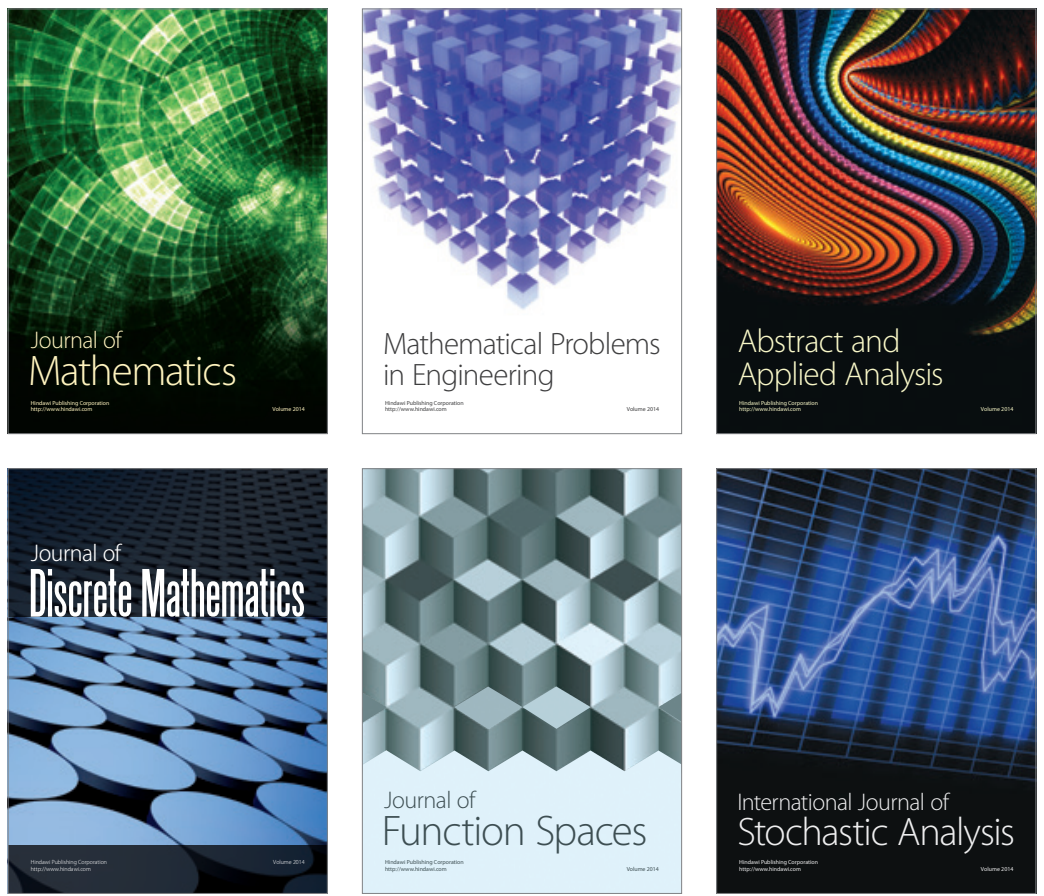

Journal of

Function Spaces

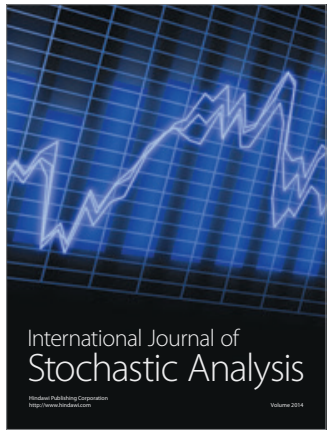

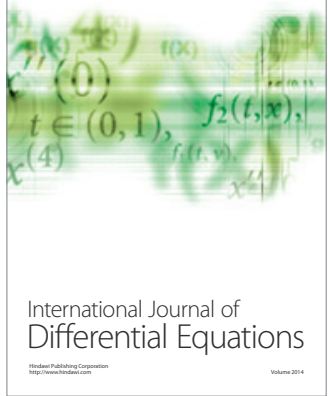
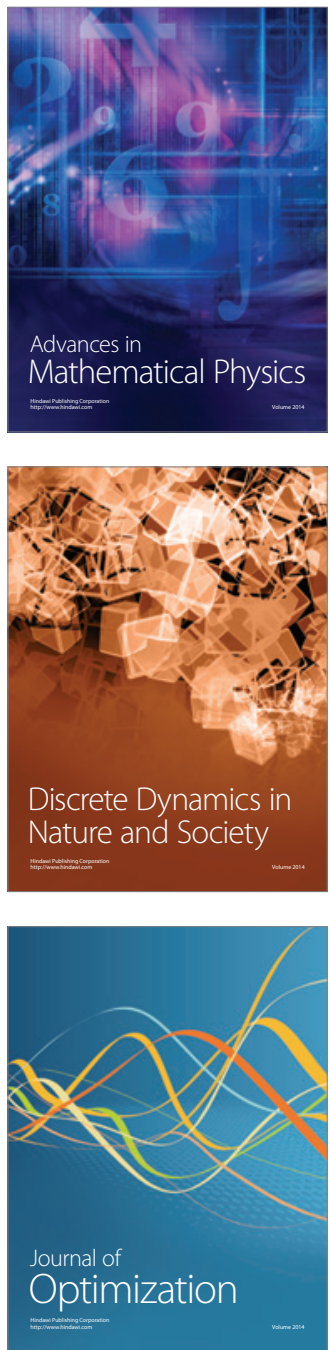